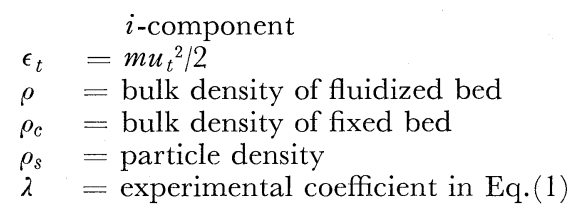

Literature Cited

1) Andrews, M.: Ind. Eng. Chem., 52, 85 (1960)

2) Blyakher, I. G. and V. M. Pavlov: Intern. Chem. Eng., 6, No. 1, 47 (1960) $\left[\mathrm{g} \cdot \mathrm{cm}^{2} / \mathrm{sec}^{2}\right]$ $\left[\mathrm{g} \cdot \mathrm{cm}^{2} / \mathrm{sec}^{2}\right]$ $\left[\mathrm{g} / \mathrm{cm}^{3}\right]$ $\left[\mathrm{g} / \mathrm{cm}^{3}\right]$ $\left[\mathrm{g} / \mathrm{cm}^{3}\right]$

\title{
ANALYSIS OF THE DISPERSING PROCESS OF LIQUID- LIQUID DISPERSED SYSTEM BY USE OF A NEW SMALL TYPE OF HIGH-FREQUENCY INDUCTANCE TORQUE METER*
}

\author{
KAZUO NAKADA AND MASATO TANAKA \\ Department of Chemical Engineering, Faculty of Engineering, \\ Niigata University, Nagaoka, Japan
}

\begin{abstract}
Using a new type of torque meter with an extremely high sensitivity, some attempts have been made to get more detailed information than ever about coalescence and dispersion in a stirred liquid-liquid system. A dimensionless empirical equation could be derived relating the perfect-dispersing time, defined from the transient characteristics of torque received by a stirrer's shaft, to the several representative dimensionless groups. Furthermore it was made clear how much portions of the entire energy given to the liquid-liquid system are directly responsible for the dispersing process itself.
\end{abstract}

\section{Introduction}

In common with the role of the temperature of the environment in the case of usual chemical reaction, even in the case of droplet interactions in a stirred tank the turbulent-field strength may be regarded as a very important factor controlling the rates of

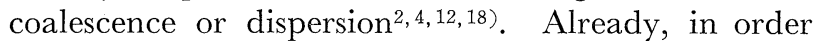
to make clear the role of turbulent energy in a stirred vessel both experimentally and theoretically, various attempts have been made from different standpoints ${ }^{5}$, $9,10)$.

However, little work has yet been undertaken to elucidate in a quantitative sense in what manner the mixing energy furnished by a rotating impeller contributes to the dispersing process, and also to what extent the same would be consumed due to the viscous friction within liquids. The one purpose of the present study was numerical estimation of the contribution of mixing energy to the dispersing process

* Received on April 5, 1971

Presented at Tohoku Meeting, Yamagata, September, 1970 and the 36th Annual Meeting of the Society of Chemical Engineers, Japan, April 1971 and the establishment of a correlation between operating conditions and perfect-dispersing time. For this purpose, authors utilized a small new type of highsensitive torque meter attachable to a low-power motor.

The slip-ring part and the pick-up part of this transducer are designed to be relatively small as compared with those of the usual torque meter commercially available. By measuring exactly the mixing torque acting on the impeller shaft, it was made clear that on the one hand the entire energy necessary for agitation was consumed to give rise to the motion of fluids and eventually to overcome the viscous friction within the liquid, and on the other hand the same was changed to the mechanical friction energy generated in the apparatus (principally in the ball-bearing support). The energy consumption due to the viscous friction within the fluid could be estimated as compared with that obtained by measuring the actualtemperature rise of the agitated liquid under adiabatic conditions. As for the liquid-liquid dispersed system, the same procedure was used to determine what portion of the mixing energy given to the fluid would be consumed to produce the dispersed phase.

Moreover, attempt were made to relate the perfect-dispersing time, which was newly defined 


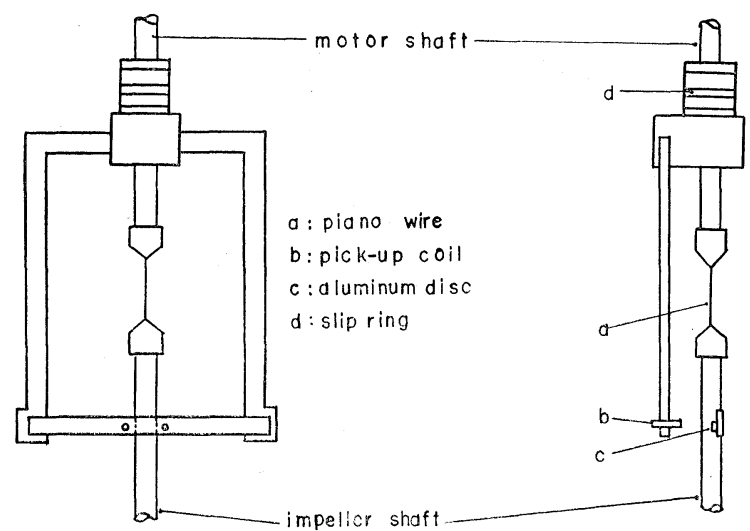

Fig. 1 Main part of torque meter

as the time required for dynamic equilibrium to be reached concerning torque variations, to the operating conditions. Consequently, an empirical equation correlating the perfect-dispersing time with the operating conditions could be derived.

\section{Experimental Apparatus}

\section{1-2 Torque meter}

A new type of sensitive torque meter capable of measuring the very low-level energy consumption of agitation was devised on the basis of the principle of high-frequency inductance-displacement meter. The main part of the torque-detecting device attached to the impeller shaft, is illustrated in Fig. 1. The impeller shaft is cut into two pieces, which are connected with each other by a thin piano wire that twists when subjected to any weak torque. The upper part of the shaft connected with the driving motor shaft, is provided with two high-frequency inductance pick-up coils (ca. $20 \mu \mathrm{H}$ ) placed on two symmetrical portions with respect to the shaft axis. The lower part of the shaft having a six-bladed turbinetype impeller is also provided with two aluminum discs for giving the inductance variation of the pick-up coils.

Both the two pick-up coils and the two aluminum discs are placed face to face, respectively, in keeping a narrow clearance (ca. $5 \mathrm{~mm}$ ) between them. The change of this clearance caused by the change of torque may result in a change of inductance in the pick-up coils and eventually bring about a change in the rectified direct-current output.

The electrical circuit of the transducer composed of the oscillator (ca. 1 Mc) part and the rectifier part is shown in Fig. 2, where a new circuit utilizing a highfrequency differential transformer is adopted instead of the phase-sensitive detector currently used. The diameter of the shaft-connecting wire, upon which the sensitivity of the torque meter essentially depends, was set to an appropriate value according as the range over which the torque received by the impeller shaft was expected to vary with the kinds of liquids tested. For example, in the case of wire diameter a) Schematic view of the whole circuit

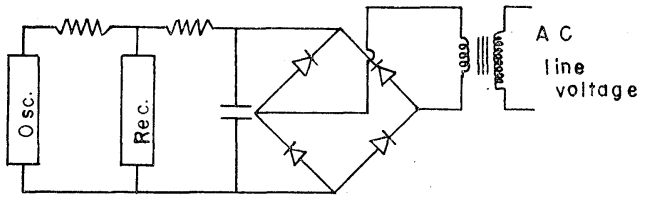

b) Oscillator part

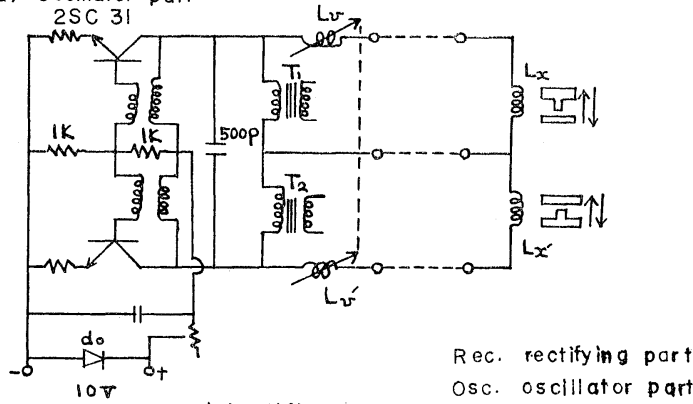

c) Rectifiar port

$T_{1}, T_{2}$. high-frequency transformer

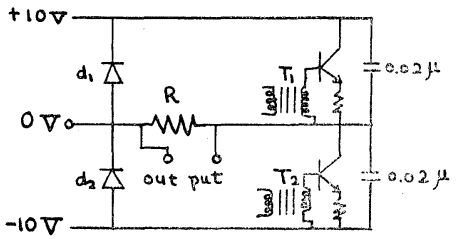

Lv, Lu. variable high-frequency
inductance

$L_{x}, L_{x}$ pick-up coil inductance

$d_{1}, d_{1}, d_{2}$ Zener diode

$R$ constunt resistance for getting rectified voltages

Fig. 2 Electrical circuit of transducer

of $1.0 \mathrm{~mm}$ allowable torque variation ranges from 0 to $826 \mathrm{~g}-\mathrm{cm}$, the resolution is $8 \mathrm{~g}-\mathrm{cm}$, and accordingly the accuracy attainable for a relatively large change of torque is ca. $1.0 \%$.

As a rotating agitator Chemi-Stirrer B-100 Type (Tokyo Rika Kikai Go. Ltd.) is used, the impeller speeds of which can be fixed at any one of sixteen definite stages over the range from 7 to 1560 r.p.m. by changing the gear ratio.

2-2 The normal stirred vessel and the adiabatic vessel

As the apparatus for studying the perfect-dispersing time and the power consumption three different sizes of baffled cylindrical-glass vessel were prepared, provided with three sizes of six-bladed turbine-type impellers.

The actual dimensions of three vessels and three impellers are shown in Table 1. The impeller diameter is equal to one-half of the vessel diameter. The four pieces of baffles, having a blade width of onetenth the tank diameter are attached to the wall of the vessel so as to take symmetrical positions. The vessels are made geometrically similar to each other, and their respective imepllers also have a geometrically similar shape.

The adiabatic vessel is used for measuring the temperature rises of the liquid mixtures, the origin of which may be regarded as the thermal energy evolved as a result of the internal viscous friction within liquids. A small Dewar vessel $(10.0 \mathrm{~cm}$ in inner diameter), commercially available, is adopted as a stirred vessel capable of retaining an adiabatic condition constant. Four baffles with a width of one- 


\begin{tabular}{|l|c|c|c|c|}
\hline & $D$ & $L$ & $d$ & $z$ \\
\hline small size & 100 & 150 & 50 & 10 \\
\hline middle size & 150 & 200 & 75 & 15 \\
\hline large size & 200 & 300 & 100 & 20 \\
\hline
\end{tabular}
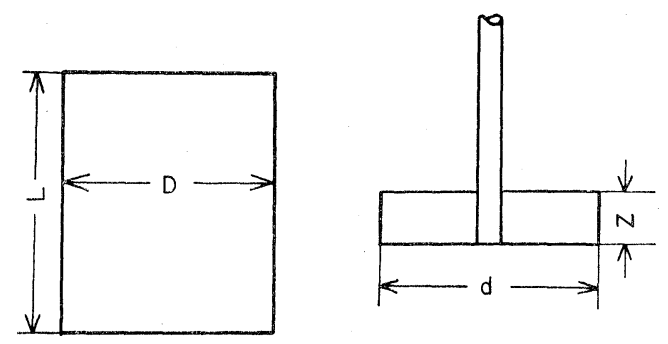

Table 1 Vessels and impellers used

tenth the vessel diameter are again fitted on to the Dewar vessel wall. The extremely small variation of liquid temperature can be detected by use of a glass thermistor probe (Toa Electronics Ltd., TDB0.1 Type), to follow which a pen recorder (Toa Electronics Ltd., EPR-2T) is used in conjunction with an amplifier (Toa Electronics Ltd., Prebox PB-11 Type). A schematic diagram of the apparatus for measuring the liquid temperature rise is shown in Fig. 3.

\section{Experimental Procedures and Conditions}

\section{3-1 Measurements of the torque for mixing and the perfect-dispersing time}

With regard to one-phase system, measurements of the torque for mixing or the power consumption are made using only the smallest stirred vessel. Aqueous glycerine solutions used as a test liquid are prepared by diluting glycerine with water to concentrations of $10,30,50$ and 70 vol. percent and viscosities of 1.1, $2.1,6.05$ and 22.8 c.p., respectively.

At the each run the vessel is filled with the same volume $(800 \mathrm{cc})$ of the diluted glycerine solution.

The impeller is always situated at one-third of the liquid depth from the bottom of the vessel. In the case of a liquid-liquid dispersed system, experiments for measuring the power consumption or the perfectdispersing time are conducted using the three geometrically similar vessels. As the continuous phase a mixture of benzene and monochlorobenzene (mixing ratio equal to $4 / 5)$ is adopted. The continuousphase liquids are prepared beforehand so as to have a density differing slightly from that of the dispersed phase for the purpose of getting easy dispersion. Unlike the case of one-phase system, the various concentrations of glycerine solutions are this time used as the dispersed phase. The same kind of run is done with respect to three different ratios of the

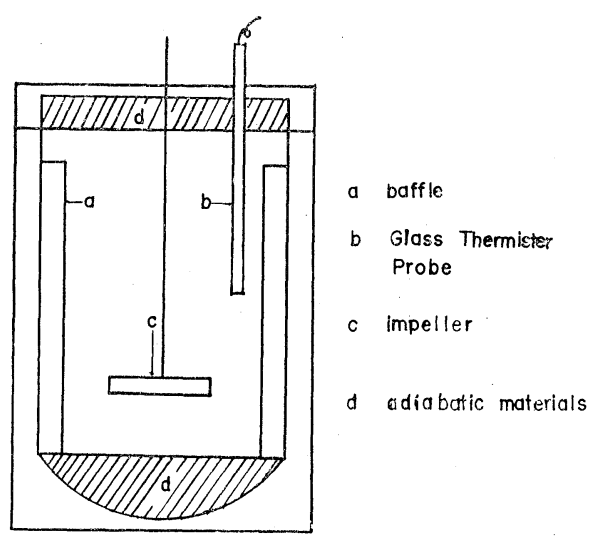

Fig. 3 Apparatus for measuring the liquid temperature rise

dispersed-phase volume to the continuous-phase volume, namely, $1 / 3,3 / 5$ and $3 / 13$. In these experiments on the liquid-liquid dispersed system the impeller is placed just above the interface, and yet not touching the interface, before agitation is started.

The impeller speeds are changed stepwise through 196, 313, 324, 512 and 521 r.p.m.

Since Reynolds numbers corresponding to these impeller speeds range from $3.2 \times 10^{3}$ to $8.7 \times 10^{4}$, it can be said that the experiments are performed in the region of turbulent field.

3-2 Measurements of temperature rises of agitated liquids under adiabatic conditions

Next, let us deal with the case where the Dewar bottle, adiabatically isolated from the surroundings, is employed as a stirred vessel. Simultaneous following of both the temperature rises and the torque variations accompanying the mixing operation is performed by use of a two-pen recorder (Toa Electronics Ltd. ERP-3T). Glycerine solutions, having the same concentrations as those mentioned just above, are used as test liquids in order to know the influence of viscosity differences of the mixed liquids on the temperature rise. The vessel is again filled with a constant volume $(800 \mathrm{cc})$ of test liquids. An inevitable, though slight, effect of heat transfer on the liquid temperatures, which seems to originate in the incompleteness of the adiabatic conditions, is checked by repeating runs at several room temperatures. Owing to the systematic check of the various temperature-rise trends obtained under different conditions of the surroundings, the undesirable effect of heat transfers could be estimated to some extent. To evaluate the energy consumption from the data on temperature rises, calibration curves capable of relating both quantities need to be constructed.

For this purpose a definite amount of heat energy is supplied to the test liquid by charging a definite intensity of electric current through heater wire $(15 \Omega)$ immersed in the liquid. Temperature rises due to electrical heating are measured while continuing very weak agitation so as to uniformly distribute this generated heat. Temperature rises due to this weak agitation were negligible as compared with 


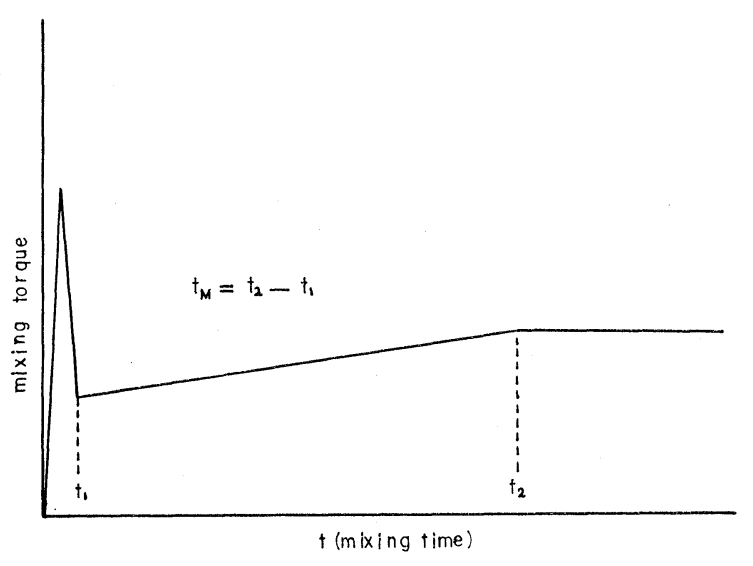

Fig. 4 Schematic picture of the variation of torque

those due to electrical heating. By comparing the rates of temperature rise originating in the agitation itself with those predicted from the calibration curve, a reliable estimation of the energy consumption associated with viscous friction emerging within liquids could be achieved.

\section{Experimental Results and Discussions}

4-1 The correlation between the operating conditions and the perfect-dispersing time

Though the dispersing process observed in the stirred vessel is extremely complex ${ }^{3,13)}$, it is obvious that their rates depend strongly on the turbulent energy of agitated liquids, aside from effects due to a few physical properties such as viscosity, density differencies of the two phases and so on. The correlation relating the dispersing processes to the operating conditions controlling these processes have not yet been presented. To make this problem clear, the experiments are done under such a condition that the more viscous liquid (glycerine solutions) is dispersed into the continuous-phase liquid (organic solvents) of the less viscous fluid.

It appears that this operation is accompanied by a relative increase in viscosity of the liquid-liquid mixture. This increase of viscosity should lead to an increase in the torque acting on the impeller shaft, which is to be observed during the course of the perfect-dispersing time. This perfect-dispersing time is denoted as $t_{2}-t_{1}=t_{M}$, as shown in Fig. 4. Bearing these considerations in mind, let us assume that the correlation which should hold between the perfectdispersing time and a few important dimensionless groups (Reynolds, Froud and Weber number) can be expressed as follows:

$$
R=t_{M} N=A\left(N d^{2} \rho / \mu\right)^{\alpha}\left(N^{2} d / g\right)^{\beta}\left(\sigma N^{2} d^{3} / \mu\right)^{r}
$$

where the dimensionless perfect-dispersing time $R$ is set equal to the product of the perfect-dispersing time $t_{M}$ and the impeller speed $N$. Since the geometrical similarity is almost completely retained among the sizes in quesion, namely the vessel height, the vessel diameter and the impeller dimension, it can be

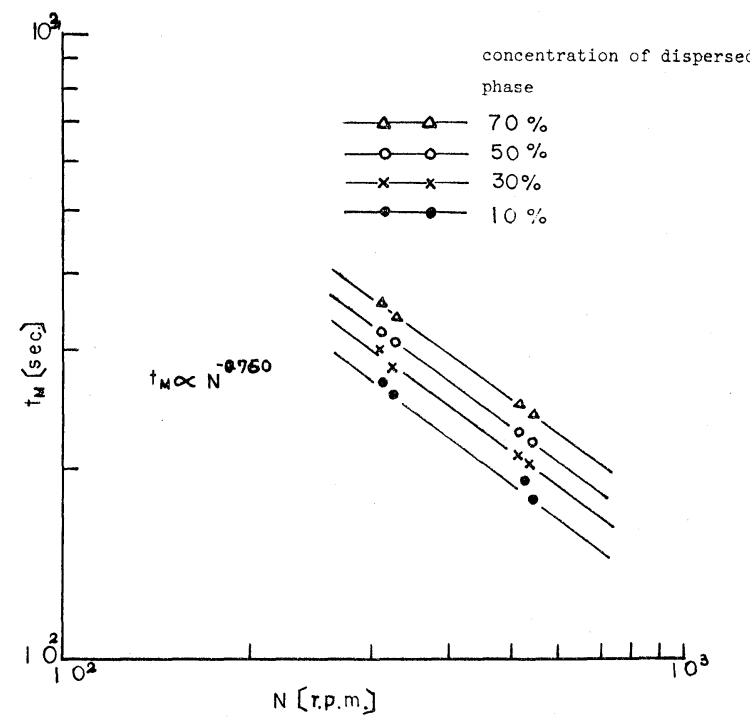

Fig. 5 Correlation between the perfect-dispersing time and the impeller speed

determined from experimental results whether Eq.(1) is acceptable or not. To determine the three exponents, $\alpha, \beta$ and $\gamma$, first the correlation between the perfect-dispersing time $t_{M}$ and the impeller speed $N$ is examined in such a manner as shown in Fig. 5.

In Fig. 5 it is demonstrated that $t_{M}$ is proportional to $N^{-0.760}$. Consequently, the correlation among these exponents in Eq.(1) can be expressed as follows:

$$
\alpha+2 \beta+2 \gamma=0.240
$$

After eliminating $\beta$ from Eqs.(1) and (2), $R F r^{-0.120}$ is plotted against $(W e / F r)$ in order to determine $\gamma$. From the slope of this plot $\gamma$ is estimated as -0.650 .

Next, upon $R e \cdot F r^{-1 / 2}$ being plotted against $R \cdot F r^{-0.120}$ $(W e / F r)^{0.650}, \alpha$ can be estimated as 0.732 . When these values of $\alpha$ and $\gamma$ are taken, $\beta$ is calculated as 0.404 from Eq.(2). Furthermore, $A$ in Eq. (1) is found to be equal to 8.47. Accordingly, the dimensionless perfect-dispersing time can be expressed in terms of the representative dimensionless groups as follows:

$$
R=8.47 \operatorname{Re}^{0.732} \mathrm{Fr}^{0.404} \mathrm{We}^{-0.650}
$$

\section{4-2 Experiments in the adiabatic stirred vessel}

In the case where mixing of the liquids was effected by agitation, the mechanical energy given to the fluid from outside must be consumed as kinetic energy in the fluid and ultimately as the thermal energy due to viscous friction within the liquid $7,8,11,14,17)$. If the adiabatic conditions of agitated systems are completely retained, the rates of temperature rise due to agitation should accord with those caused by the electrical heating required for calibration. Taking these facts into consideration, mixing was conducted by adopting an aqueous solution of glycerine as the test liquid. As a result of experiments the linear correlation between temperature rise and time can be established as shown in Fig. 6. From the rate of temperature rise the energy consumption $(m W)$ can be estimated on the assumption that the measured 


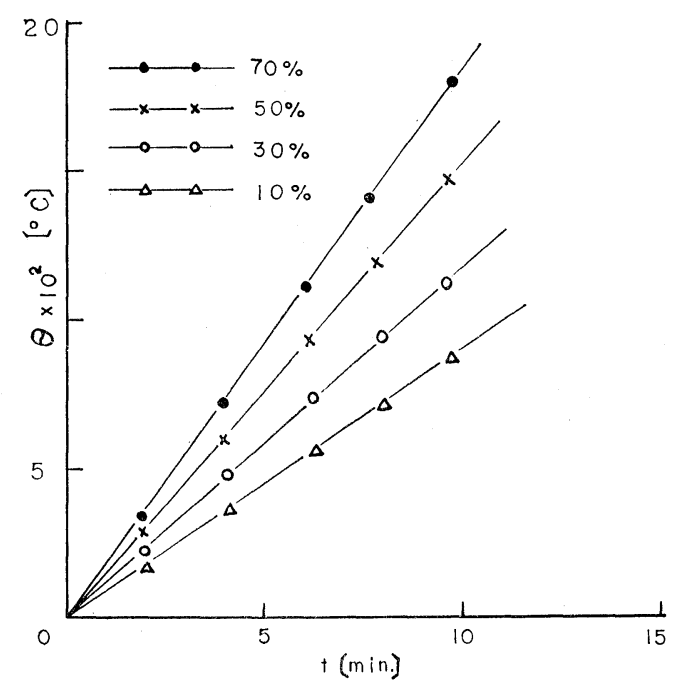

Fig. 6 Correlation between the temperature rise and the time

$$
\text { (at } 521 \text { r.p.m.) }
$$

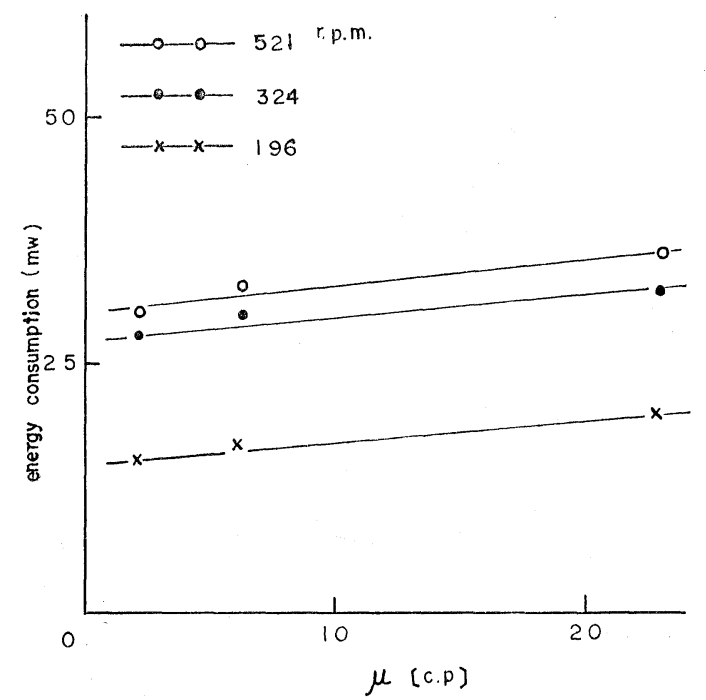

Fig. 7 Plots of the energy consumption lost within the liquid vs. the viscosity of the liquid

rate of temperature rise, $d \theta / d t$, is equivalent to that constructed for calibration. It is obvious that this energy consumption should be considered as the energy dissipation due to viscous friction. To make clear the extent to which the energy consumption depends on the viscosity of agitated liquids, a plot of energy consumption against viscosity is made as shown in Fig. 7.

From Fig. 7 the numerical estimation of the energy consumption associated with viscous friction within the fluid can be readily made, while the entire energy consumption for agitation is known from the mixing torque measured simultaneously. From the above consideration the energy consumption for agitation can be divided into the energy consumption due to the mechanical friction of the ball-bearing support and the energy actually consumed by viscous friction.

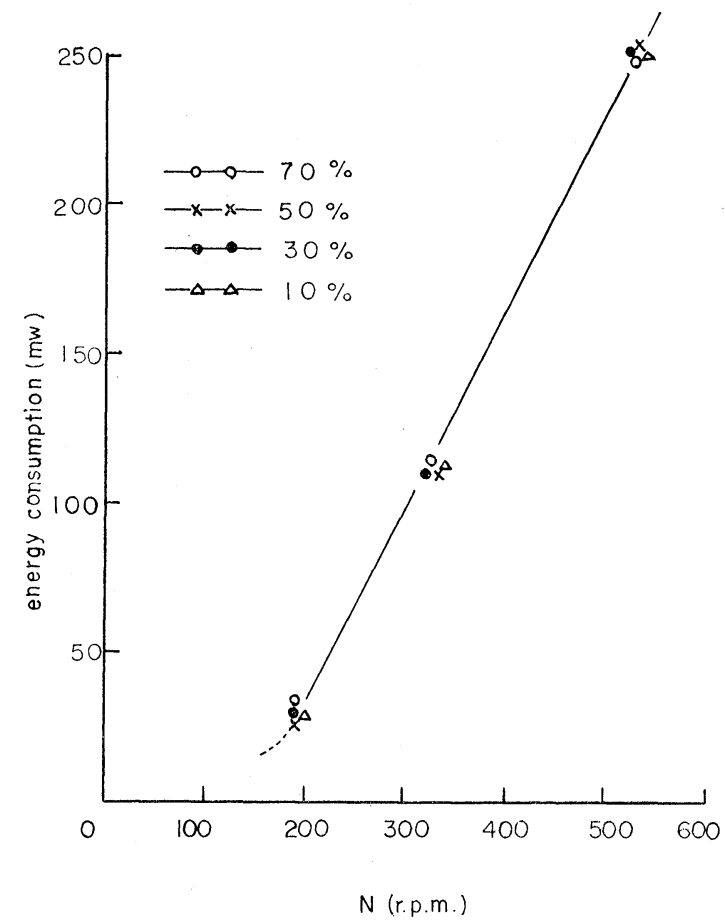

Fig. 8 Plots of the energy consumption due to the mechanical friction vs. the impeller speed

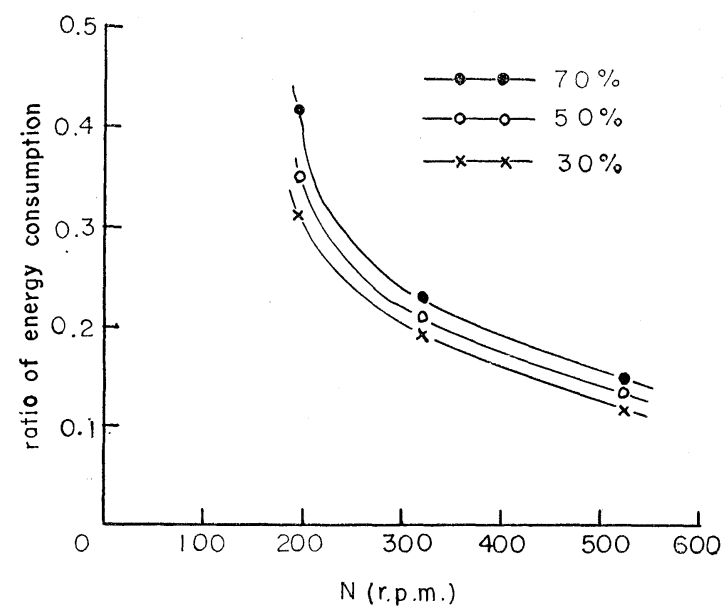

Fig. 9 Plots of the ratio of the energy consumption to the viscous friction to the energy consumption vs. impeller speed

In view of these situations the conclusion is drawn that the values of energy consumption obtained by the subtraction of the energy consumption due to viscous friction from the entire energy consumption for agitation, must be equal to the energy consumption due to the mechanical friction inherent in the apparatus itself. The energy consumption calculated in this way must be constant so long as the impeller speed is the same, even though the test liquids are different. Actually, as shown in Fig. 8, where the calculated power requirements directly ascribable to mechanical friction alone are plotted against impeller speed, almost all points are found to lie close to only the one curve in spite of the different viscosity 


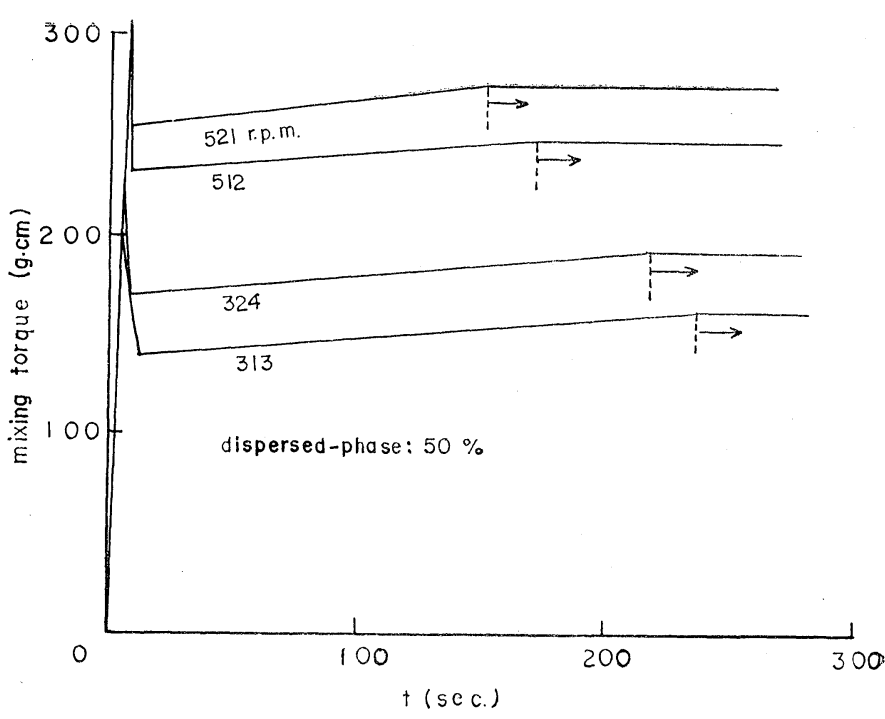

Fig. 10 The transient process of the mixing torque varying with the time

of test liquids, namely the different concentration of glycerine solution*.

Accordingly, it is possible to say that the measured values of energy consumption due to viscous friction within the fluid are reliable. Next, to make clear what portion of the entire energy for agitation is given to the fluid and finally converted into thermal energy on account of the viscous friction, the ratio of the energy consumption is plotted against impeller speed in Fig. 9. From Fig. 9 it is readily noticed that 10 to 40 percent of the energy consumption for agitation was expended due to viscous friction within the liquid.

\section{4-3 Experiments in a normal stirred vessel}

To know what portion of the total mixing energy given to the fluid contributes to the dispersing process for producing the dispersed system, a series of experiments were conducted using a normal stirred vessel.

For this purpose only the smallest vessel, namely, $10 \mathrm{~cm}$ in diameter and $15 \mathrm{~cm}$ in height, is used. Since the impeller is placed just above the interface before agitation is initiated, the transient process of the mixing torque must pursue the same course as that mentioned in 4-1. The transient variation of the torque measured in this way is shown in Fig. 10. In view of this figure, the power consumption required for the final equilibrium state to be reached appears to correspond to the energy consumption necessary for mixing of the continuous phase and the dispersed phase to produce an emulsified state. To know the extent of the contribution of the mixing energy

* The reason why the approximately linear plot of torque due to the mechanical friction against impeller speed does not pass through the origin in Fig. 8 must be referred to. Contrary to the case exceeding ca. 100 r.p.m., in the case from 0 to ca. 100 r.p.m. definite values of torque are difficult to obtain because of the ambiguous contribution of static friction that is sure to prevail over the dynamic friction.

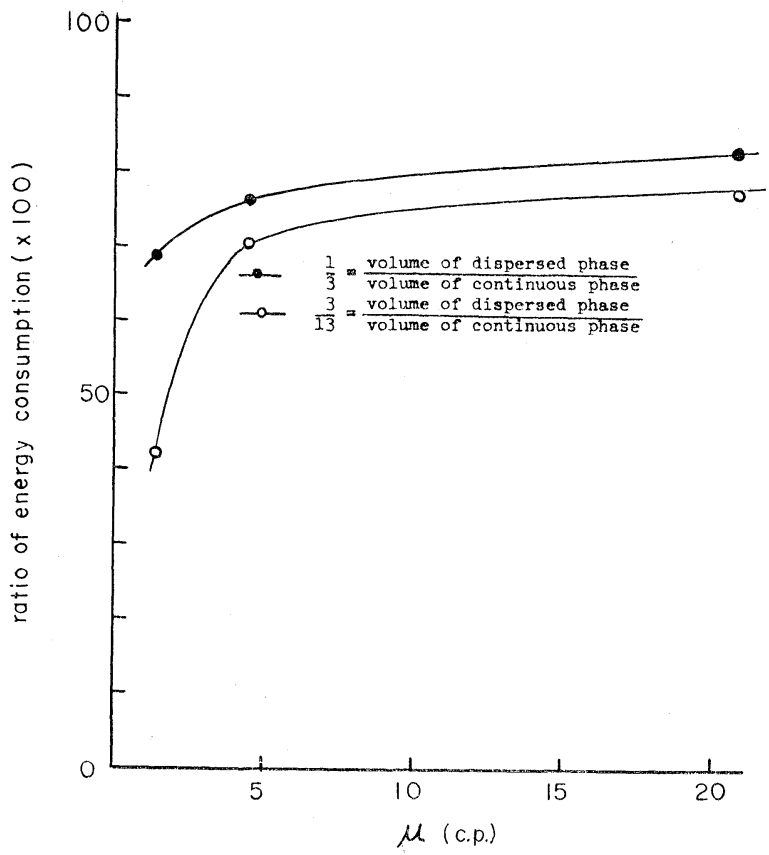

Fig. 11 Plots of the ratio of the energy consumption required to produce the dispersed droplets to the total energy consumption against the viscosity of the dispersed phase

given to the liquid-liquid system to the dispersing process alone, the ratios of the energy consumption expended to produce the dispersed droplets to the total energy consumption for executing the mixing are plotted against the viscosity of the dispersed phase as shown in Fig. 11. If the energy dissipation in the continuous and in the dispersed phase were assumed to be equal, the energy consumption due only to the dispersing process of the dispersed-phase liquid is obtained by deducting the energy consumption for mixing the continuous phase alone from the entire energy consumption lost during the perfectdispersing time.

From Fig. 11 it is noticed that in the case where the two-phase system is dealt with, 40 to 80 percent of the energy associated with liquid mixing was found to be responsible for the dispersion itself.

\section{Conclusion}

Investigations of the contribution of the mixing energy to the energy required for dispersing were conducted by use of a small new type of high-sensitive torque meter developed by the authors. Main conchusions drawn can be summarized as follows:

1) The empirical equation relating the operating conditions to the perfect-dispersing time are expressed in the following form:

$$
R=8.47 \operatorname{Re}^{0.732} \mathrm{Fr}^{0.404} \mathrm{We}^{-0.650}
$$

2) With regard to the present apparatus it was shown that only 10 to 40 percent of the entire energy consumption for agitation was given to the agitated liquid. It was also demonstrated that 40 to 80 percent of the total energy received by the agitated liquid was spent to produce the dispersed state until the 
dispersing process had been completed.

\section{Acknowledgement}

The authors express their thanks to Mr. H. Hiura and Mr. T. Yamada for their experimental cooperation in the course of this study.

\section{Nomenclature}

$d=$ impeller diameter

$D=$ vessel diameter

$H=$ liquid depth

$N=$ impeller speed

$R=$ dimensionless perfect-dispersing time

$t=$ time

$t_{M}=$ perfect-dispersing time

$\alpha, \beta, \gamma=$ exponents of dimensionless groups

$\theta=$ temperature rise

\section{Literature Cited}

1) Biggs, R. D.: A.I.Ch.E.J., 9, 636 (1963)

2) Groothuis, H. and F. J. Zuiderweg: Chem. Eng. Sci., 19, 63 (1964)

3) Garl, M. W. and E. L. Gerden: Chem. Eng. Prog., 51,
85 (1955)

4) Howarth, W. J.: A.I.Ch.E.J., 13, 1007 (1967)

5) Howarth, W. J.: Chem. Eng. Sci., 19, 33 (1964)

6) Holmes, D. B., R. M. Voncken and J. A. Dekker: ibid., 19, 201 (1964)

7) Chen, H. W. and S. Middleman: A.I.Ch.E.J., 13, 989 (1967)

8) James, M. C. and R. Shimer: Ind. Eng. Chem., 53, 479 (1961)

9) Karan, H. J. and J. C. Bellinger: I \& E C. Fund., 7, 576 (1968)

10) Kenneth, J. V., Oleghbilous and N. R. Ammundson: ibid., 4, 271 (1966)

11) Kin, W.J. and F. S. Manning: A.I.Ch.E.J., 10, 747 (1964)

12) Madden, A. J. and L. Damerell: ibid., 8, 233 (1962)

13) Novak, V. and F. Rieger: Trans. Inst. Chem. Engs. (London), 47, 335 (1969)

14) Philip, L. F. and R. L. Bates: A.I.Ch.E.J., 9, 338 (1963)

15) Rushton, J. H., E. W. Costich and J. H. Everett: Chem. Eng. Progr., 46, 395 (1950)

16) Rogert, J.: Ind. Eng. Chem., Process Design and Develop, 6, $340(1967)$

17) Sprow, F. B.: Chem. Eng. Sci., 22, 435 (1967)

18) Tanaka, M., Nakada, K. and Y. Oyama: Kagaku Kögaku., 34, 893 (1970)

\title{
RESIDENCE TIME DISTRIBUTION OF SOLIDS IN STIRRED-FLUIDIZED AND MOVING BEDS*
}

\author{
SETSUJI TONE, ISAO ODA**, KOJI KAWAMURA*** \\ AND TSUTAO OTAKE \\ Department of Chemical Engineering, Osaka University, Toyonaka, Japan
}

\begin{abstract}
The flow pattern of solids within stirred beds in batch operation was studied by following tracer particle movement in a cylindrical tube. In addition, in stirred-fluidized and moving beds with continuous flow of solids, the F-curve to a step input of a tracer was determined by the electric capacitance method under various conditions. From the observation of solids movement in a stirred bed in batch operation and characteristics of the F-curves, a flow model for the stirred moving bed was developed by considering a plug flow zone, two perfect mixing zones and a dead space zone with a circulation of solids within the beds, and the F-function was derived according to the flow model. The model parameters are evaluated by matching the $F$-function to the experimental data, and the effect of operating conditions on the model parameters is discussed.
\end{abstract}

\section{Introduction}

For the operation and design of gas-solid fluidized and moving bed reactors, several studies of solids mixing have been reported. Tailby and Cocquerel ${ }^{3)}$ studied the effect of operating conditions on solid mixing in fluidized beds with continuous flow of solids and analyzed the experimental $F$-curve using the

* Received on May 21, 1971

Presented at the 3rd Autumn Meeting of the Soc. of Chem. Engrs., Japan (Osaka, Oct. 15, 1969)

** Mitsubishi Chemical Co., Ltd.

*** Asahi Chemical Co., Ltd. two quantities of holdback and segregation, as proposed by Danckwerts ${ }^{1}$. Morris et al. ${ }^{2)}$ reported that the residence time distribution (RTD) of solids in fluidized beds is not described by a simple diffusional model, but may be treated by the perfect mixing system in which some solid-bypassing occurs. Wolf and Resnick $^{5)}$ proposed an analytical method for specifying the RTD curve with two model parameters. Recently, Yano et al. $\left.{ }^{6}\right)$ analyzed the $F$-curve in the stirred-moving bed by introducing a cross-flow model between solids in the stagnant zone and active solids passing through beds. The author and coworkers ${ }^{4)}$ previously studied the mutual relation- 